\title{
BMJ Open Exploring pain interference with motor skill learning in humans: a protocol for a systematic review
}

\author{
David Matthews (D) , ${ }^{1}$ Edith Elgueta Cancino (D) , ${ }^{1}$ Deborah Falla (iD) , ${ }^{1}$ \\ Ali Khatibi (1) ${ }^{1,2}$
}

To cite: Matthews D, Elgueta Cancino E, Falla D, et al. Exploring pain interference with motor skill learning in humans: a protocol for a systematic review. BMJ Open 2021;11:e045841. doi:10.1136/ bmjopen-2020-045841

- Prepublication history and additional supplemental material for this paper are available online. To view these files, please visit the journal online (http://dx.doi.org/10.1136/ bmjopen-2020-045841)

Received 14 0ctober 2020 Accepted 23 June 2021

Check for updates

(c) Author(s) (or their employer(s)) 2021. Re-use permitted under CC BY-NC. No commercial re-use. See rights and permissions. Published by BMJ.

${ }^{1}$ Centre of Precision Rehabilitation for Spinal Pain (CPR Spine), School of Sport, Exercise and Rehabilitation Sciences, University of Birmingham, Birmingham, UK ${ }^{2}$ Centre for Human Brain Health, University of Birmingham, Birmingham, UK

Correspondence to David Matthews; dxm986@student.bham.ac.uk

\section{ABSTRACT}

Introduction Motor skill learning is intrinsic to living. Pain demands attention and may disrupt non-pain-related goals such as learning new motor skills. Although rehabilitation approaches have used motor skill learning for individuals in pain, there is uncertainty on the impact of pain on learning motor skills.

Methods and analysis The protocol of this systematic review has been designed and is reported in accordance with criteria set out by the Preferred Reporting Items for Systematic Review and Meta-Analysis Protocols guidelines. Web of Science, Scopus, MEDLINE, Embase and CINAHL databases; key journals; and grey literature will be searched up until March 2021, using subjectspecific searches. Two independent assessors will oversee searching, screening and extracting of data and assessment of risk of bias. Both behavioural and activitydependent plasticity outcome measures of motor learning will be synthesised and presented. The quality of evidence will be assessed using the Grading of Recommendations Assessment, Development and Evaluation approach. Ethics and dissemination No patient data will be collected, and therefore, ethical approval was not required for this review. The results of this review will provide further understanding into the complex effects of pain and may guide clinicians in their use of motor learning strategies for the rehabilitation of individuals in pain. The results of this review will be published in a peer-reviewed journal and presented at scientific conferences.

PROSPERO registration number CRD42020213240.

\section{INTRODUCTION}

In 2020, the International Association for the Study of Pain (IASP) revised its definition of pain to reflect the progress made over the last thirty years around the understanding of pain. The new definition states that pain is 'an unpleasant sensory and emotional experience associated with, or resembling that associated with, actual or potential tissue damage'. ${ }^{1}$ The new definition clearly notes that pain is a "personal experience that can be influenced by varying degrees by biological, psychological and social factors'.

The experience of pain is considered primarily protective; it is perceived as aversive

\section{Strengths and limitations of this study}

This is the first systematic review synthesising evidence exploring pain interference with motor learning in humans.

- The design of this study follows the recommendations laid out in the Preferred Reporting Items for Systematic Review and Meta-Analysis Protocol guidelines.

- The meta-analysis will include only low and moderate risk-of-bias studies, assessed using appropriate risk-of-bias tools, for both randomised control and non-randomised studies.

- To provide consistency in reporting results, the Grading of Recommendations Assessment, Development and Evaluation approach will be used.

- Due to the potential for large methodological and clinical heterogeneity of the included studies, subgrouping will be explored to ensure useful conclusions for researchers and clinicians.

and motivates individuals to act. ${ }^{2}$ Such action disrupts goal-orientated behaviour. ${ }^{3}$ For example, pain-related goals, such as seeking relief, can conflict with non-pain goals, ${ }^{4}$ such as learning a new skill or using an already acquired one. In short-lasting pain, brief disruption of functional goals is seen as beneficial or protective and is considered to have little impact on learning or memory of the disrupted functional goals.

In some cases, the presence of an ongoing perceived threat, despite the defensive action of a brief disruption of functional goals, results in persistent protective behaviour and persistence of a pain experience. ${ }^{2}$ Such persistent pain is the leading cause of disability according to the 2016 Global Burden of Disease review. ${ }^{5}$ In individuals with persistent pain, prolonged defensive action and interruption of functional tasks may limit encoding of activity and task-related information into memory. ${ }^{6}$ Research has consistently demonstrated activities are performed with 
less accuracy and more slowly after being interrupted by pain. $^{7}$

Pain disrupts the motor system at many levels. ${ }^{8}$ There is a wealth of behavioural and neurophysiological evidence that pain effects the motor system. ${ }^{9}$ One such change is an alteration in the ability of the motor system to adapt to repeated skilled practice associated with impaired skill performance. ${ }^{10}$ Boudreau and colleagues ${ }^{10}$ demonstrated reduced motor performance following motor skill learning in the presence of pain and reduced motor cortex excitability in the primary motor cortex, a measure associated with cortical plasticity. ${ }^{11}$

In contrast to the above, research demonstrating the neural substrate for such an interaction is less conclusive. Neuroimaging studies have identified a cerebral signature of pain, ${ }^{12}$ including areas associated with motor planning and execution, such as the anterior cingulate cortex, premotor and primary motor cortex, cerebellum and basal ganglia. ${ }^{13}{ }^{14}$ Misra $(2015)^{15}$ reported an increase in blood oxygenation level dependent (BOLD) activity in the midcingulate cortex in response to pain or movement and when they occurred simultaneously. In the same research group, Coombes $(2016)^{9}$ identified areas of the cerebellum (left lobules VI and VIIb) that demonstrate overlapping roles during motor activity and pain and continue to be active in the presence of both. Both these areas have been associated with motor adaptation and have anatomical and functional connections with the motor cortex. ${ }^{16}$ Connections from the striatum (basal ganglia) and the cerebellum to the motor cortex have been found to play a key role in early stages of motor skill learning. ${ }^{17} 18$

Motor skill learning involves repeated task practice, resulting in effortless and efficient performance of a movement. ${ }^{19}$ Research has identified three stages of motor learning common across all motor learning tasks: early (acquisition), intermediate (consolidation) and late stages (retention). Early stage is within session learning, consolidation is learning that occurs offline or in between sessions and retention refers to learning across more than one session. ${ }^{18}$ Motor skill learning is intrinsic to life. Novel life experiences, such as learning to walk or drive, require adaptations of the motor system to maintain efficient interactions with the environment whilst using minimal attentional processes. Conversely, loss of function due to injury or disease requires relearning of previously wellestablished motor patterns or learning new motor skills within the limitations of function. Motor skill learning is common to many rehabilitation approaches used to help individuals manage their pain. Principles of motor skill learning applied to exercise for low back pain have been shown to reduce pain and improve muscle activity, which is accompanied by activity-dependent plasticity enhancing normalisation of networks of the primary motor cortex. ${ }^{20}$

The effectiveness of motor skill learning is commonly assessed using measures of task performance and activitydependent plasticity measures. Typical measures of postlearning task performance include the number of errors or measurement of spatial errors; measures of accuracy, such as distance away from ideal performance; or temporal measures, such as speed of performance, acceleration or reaction/response times. ${ }^{17}$ Measures exploring neural correlates related to motor learning have been used to provide further insights into processes underlying the acquisition of motor skills. ${ }^{21}$ Activity-dependent plasticity can be demonstrated using neuroimaging techniques such as functional magnetic resonance imaging (fMRI) (changes in amplitude, temporal and spatial characteristics of BOLD signals), ${ }^{22}$ transcranial magnetic stimulation (TMS) (changes in amplitude, temporal and spatial characteristics of motor evoked potentials (MEPs) and intracortical excitability ${ }^{11}$ and electroencephalogram (EEG) (changes in amplitude of somatosensory evoked potentials (SEPs) ). ${ }^{23}$ Methods of analysing movement strategies people use when learning a motor skill have included but are not limited to end point errors, motor activity using electromyography (EMG) and biomechanical analysis.

Early animal studies demonstrated impaired adaptive learning in the presences of nociception in spinalised rats. ${ }^{24}{ }^{25}$ Subsequent research exploring this phenomenon in humans has provided mixed results. A within-subject study design from $2007^{10}$ explored pain interference in the acquisition phase of motor learning during a visuomotor task. The authors demonstrated an impairment of improvements in performance behaviour following 15 min of motor skill learning in the presence of capsaicininduced pain. In contrast to the above study, Bouffard et al $(2014)^{26}$ demonstrated no impairment in acquisition of a locomotion motor skill when pain was experienced during the task. Instead, they found impairments in the retention phase of learning 48 hours after the session. Differences in results may be explained in part due to the use of a tonic pain paradigm, not influenced by engagement in the task, and the choice of a motor adaptation intervention, reportedly dependent on different neural mechanisms compared with motor sequence learning. ${ }^{27}$ Subsequent studies exploring impact of pain on behavioural measures following motor learning have demonstrated no change, ${ }^{28-31}$ or an improvement in performance. ${ }^{32-34}$

No systematic review has synthesised the evidence for the impact of pain on task performance measures and/or activity-dependent plasticity measures following motor skill learning in humans. The wide variety of motor learning paradigms, pain paradigms and outcome measures meant that comparisons and interpretation of results are not straightforward. It is possible that due to varying cognitive and attentional demands of different motor learning tasks, the interaction with pain will vary. ${ }^{35}$

The main objectives of the proposed systematic review are to (1) summarise existing literature to establish the evidence of pain interference on task performance measures following motor skill learning; (2) summarise activity-dependent plasticity measures associated with the cerebellum, corticospinal tract and primary motor area assessed in the acquired literature in response to the 
observed pain interference; (3) describe the different pain paradigms and motor skill learning paradigms used in the research to explore pain interference of motor skill acquisition and discuss how and possible reasons why the resultant interaction varies; and (4) critically evaluate the methodological quality of the studies on pain interference of measures of motor performance following motor skill learning.

\section{METHODS}

The protocol of this systematic review has been designed following a scoping literature search and is reported in accordance with criteria set out by the Cochrane Handbook and Preferred Reporting Items for Systematic Review and Meta-Analysis Protocols guidelines. ${ }^{36} 37$

\section{ELIGIBILITY CRITERIA \\ Inclusion criteria}

The participants, interventions, comparators, outcomes and study design (PICOS) framework will be used to inform the eligibility criteria for the inclusion and exclusion of studies. ${ }^{36}$

\section{Populations}

Adults (age $\geq 18$ years old) experiencing clinical or experimental pain. Including studies on both clinical and experimental pain will provide deeper insights into the interactions of pain with motor learning due to the complex nature of the pain experience. Clinical pain will be any symptoms of pain included in the IASP definition for pain mentioned above excluding those occurring in the presence of neurological disease or due to delayedonset of muscle soreness (DOMS). The location of pain will not be restricted. Confounding factors known to impact on the outcome of interventions, such as duration of pain, anxiety, depression, low mood and associated motor and sensory disturbances, will be extracted from the studies where appropriate. A control group including adults (age $\geq 18$ years old) with no pain will make up a comparison group.

\section{Intervention}

Pain during motor skill learning is the experimental condition being analysed. As mentioned above, studies using healthy subjects experiencing experimentally induced pain and studies evaluating people with clinical pain will be included. All study participants will be required to complete a motor skill learning task, with the explicit intention to improve their performance across the session.

Motor skill learning refers to 'the increasing spatial and temporal accuracy of movements with practice'. ${ }^{38}$ Implicit and explicit learning interventions consistent with definitions of motor skill learning used in the introduction of this systematic review will be the focus of this review. Interventions will involve repeated practice and are characterised either by simple repeated movements, the ability of subjects to combine isolated movements into well-rehearsed and smooth sequences (motor sequence learning, both simple and complex) or to compensate in response to a mechanical perturbation (motor adaptation). ${ }^{18}$ Prism adaptation paradigms will be excluded from this review in an attempt to reduce confounding variables, such as the impact of visual perception, focusing on the impact of pain on motor learning. Further variations in the motor learning paradigms will be extracted from the studies and included in the discussions.

\section{Comparators}

To explore the impact of pain on the outcome of motor skill learning, an appropriate comparison group is essential. Studies included in this review are required to have a 'healthy, no pain' comparison group or condition. A 'healthy, no pain' comparison group was defined as subjects with no acute or chronic pain; no history of recurrent pain; and no history of psychiatric, neurological or musculoskeletal disease or injury. The comparison group or condition will be required to complete the same motor skill intervention as the experimental group.

\section{Outcomes}

Outcome measures will include measures of task performance related to motor learning and activity-dependent plasticity measures. Measures of task performance will include the number of errors, or measurement of spatial errors; measures of accuracy, such as distance away from ideal performance; or temporal measures, such as speed of performance, acceleration or reaction/response times. Activity-dependent plasticity measures related to motor learning will be discussed if available to provide further insight into the understanding of pain interference. These may include changes in amplitude, temporal or spatial characteristics of BOLD fMRI signals, or MEP evoked by TMS, other TMS paradigms such as TMS-MEP response curves and intracortical inhibition or changes in amplitude of SEPs from EEG. A further requirement of included studies is that within-session gains have been established using appropriate data analysis of outcome measures.

\section{Study design}

Following a scoping review, randomised control studies were identified as the gold standard study design to demonstrate the impact of pain on the outcome of the intervention. Other study designs will be considered, including both within-subject and between-subject designs, provided that the interference of pain on study outcome measures following motor skill learning can be determined from the results. Clinical pain models make it difficult to randomise group allocation especially when the comparator is a healthy subject. As a result, quasiexperimental studies will be included in this review. 


\section{Study duration}

Although study duration will not be limited, stages of motor learning will be considered as a scoping review has revealed potentially different interactions of pain with motor learning depending on the stage of learning. ${ }^{10639}$ Research has demonstrated potential different neural mechanisms ${ }^{18}$ underlying the different stages of motor learning, which may influence pain interactions.

\section{Exclusion criteria}

The study involves reviewing research exploring the impact of pain on motor learning in an intact nervous system. Therefore, studies exploring populations with known neurological disorders involving the peripheral or central nervous system will be excluded. Any study including treatments as an adjunct to motor learning or using DOMS experimental pain models, will be excluded based on the challenges of differentiating the impacts of pain from the impacts of physiological processes related to involved treatments or DOMS. Single case studies, case series and review papers along with any studies not published in English will be excluded.

\section{Information sources}

Comprehensive searches of the following databases will be completed by the lead reviewer, from inception until March 2021: Web of Science, Scopus, MEDLINE, Embase and CINAHL. Hand searching of preprint repositories, including PsyArxiv and BioArxiv, will be completed followed by a screening exercise of references and citation lists from the articles, which meet the eligibility criteria. Authors' lists of eligible articles will also be explored.

\section{Search strategy}

Search strategies were designed (see online supplemental file), including MeSH terms and natural language combinations, in conjunction with a health sciences librarian and agreed by all authors. Keywords and their synonyms were identified and entered into databases using the Boolean terms AND/OR. The search process was streamlined by piloting the search strategy with MEDLINE, confirming MeSH terms and checking relevant article search terms. The strategy was adapted for use with other databases.

\section{Data management}

Articles resulting from the search process will be downloaded to EndNote (V.9 or later) software (Clarivate Analytics) and duplicates identified and deleted.

\section{Study selection}

Two reviewers (DM and EEC) will independently screen titles and abstracts against the predetermined inclusion and exclusion criteria. Studies will be categorised into include, exclude or undecided, and full articles will be downloaded for articles meeting the inclusion criteria. For clarification, full texts will be downloaded for studies where uncertainty still exists. Any disagreements will be first discussed by the two reviewers (DM and EEC), and where consensus is not reached, an independent reviewer will be consulted (AK). Once the above procedure has been completed and full texts have been collated, the screening process is repeated. Information on, and reasons for excluding studies will be reported.

\section{Data extraction}

Data extraction will be performed using a data extraction form developed from information gathered from early literature scoping activities (see table 1). The data extraction form will initially be piloted to ensure relevant data is being extracted and amendments made as appropriate prior to final data extraction. This will be completed independently by both reviewers (DM and EEC) to maintain autonomy.

\section{Data items}

Data items to be extracted are documented in table 1 . Authors will be contacted if clarity is required during extraction of data items. This could be due to missing data and ambiguity of results or to avoid duplication, that is, if more than one article is identified representing a single data set. In such cases, the lead and/or corresponding authors will be contacted by email, and a reminder will be sent 1 week later. Where the author does not respond within 4 weeks of the original email and the clarification impacts on the eligibility of the study, the study will be considered ineligible.

\section{Risk of bias}

Experimental randomised control trials (RCTs) and nonrandomised studies are likely to be included in this systematic review. The Cochrane risk-of-bias tool for randomized trials (ROB2) has been the most commonly used tool for assessing risk of bias in RCTs and is now considered the gold standard. ${ }^{40}$ Previous systematic reviews have used this same tool to assess risk of bias for non-randomised studies. Quigley et al (2019) ${ }^{41}$ reported that risk-of-bias assessments designed for RCTs were inappropriately used for non-randomised studies, but there is no consensus on the best tool for these studies. ${ }^{40}$ The risk of bias in non-randomized studies of interventions (ROBINS-I) will be used to assess risk of bias for non-randomised studies. This tool has been designed to assess risk of bias for non-randomised studies exploring the impacts of interventions and is becoming increasingly popular in recent years. ${ }^{40}$ Each study will be independently assessed by the two reviewers (DM and EEC) using the appropriate tool and risk-of-bias judgements recorded for the study overall (see tables 2 and 3). Where a consensus cannot be found, a third author (AK) will be consulted. Cohen's kappa coefficient will be calculated to explore agreement between the two reviewers.

\section{Data synthesis}

Where studies are sufficiently homogenous in populations (clinical heterogeneity) and motor learning intervention and outcome measures (methodological heterogeneity), a meta-analysis will be considered. Statistical heterogeneity 
Table 1 Overview of data items to be extracted from included studies

\begin{tabular}{|c|c|}
\hline Content & Data items \\
\hline General study information & $\begin{array}{l}\text { Authors } \\
\text { Title } \\
\text { Year }\end{array}$ \\
\hline Study characteristics & Study design, sample size (both groups), duration of follow-up. Inclusion/exclusion criteria \\
\hline Participant information & Age, gender (experimental or clinical pain group and comparison group) \\
\hline Outcome of interest & $\begin{array}{l}\text { Task performance measures (as appropriate): } \\
\text { Speed. } \\
\text { Number of errors. } \\
\text { Accuracy/error measure. } \\
\text { Reaction/response times. } \\
\text { Neural correlates (as appropriate): } \\
\text { Somatosensory evoked potentials. } \\
\text { Amplitude and temporal characteristics of motor evoked potentials (MEPs). } \\
\text { Motor thresholds. } \\
\text { Spatial characteristics of motor cortical maps } \\
\text { Transcranial magnetic stimulation-MEP curves } \\
\text { Cerebellar inhibition. } \\
\text { Short-interval intracortical inhibition. } \\
\text { Change in blood oxygenation level dependent fMRI signals (spatial and temporal). }\end{array}$ \\
\hline Results & Main findings, statistical analysis methods \\
\hline
\end{tabular}

fMRI, functional MRI.

will be assessed using the $\mathrm{I}^{2}$ statistics. Due to the heterogeneity of motor training and pain paradigms and the resulting likelihood of a range of mean effect sizes, the random-effects model will likely be more appropriate for meta-analysis. In line with recommendations, the meta-analysis will report on mean effect size and heterogeneity of effect size. ${ }^{42}$ Only 'low or moderate risk of bias' studies for non-randomised studies (ROBINS-I) ${ }^{44}$

Table 2 Interpretation of overall risk-of-bias judgements in ROBINS-I

\begin{tabular}{|c|c|c|}
\hline Judgement & Across domains & Criterion \\
\hline Low risk of bias & $\begin{array}{l}\text { The study is comparable to a well-performed } \\
\text { randomised trial }\end{array}$ & $\begin{array}{l}\text { The study is judged to be at low risk of bias for all } \\
\text { domains }\end{array}$ \\
\hline Critical risk of bias & $\begin{array}{l}\text { The study is too problematic to provide any } \\
\text { useful evidence and should not be included in } \\
\text { any synthesis }\end{array}$ & $\begin{array}{l}\text { The study is judged to be at critical risk of bias in at } \\
\text { least one domain }\end{array}$ \\
\hline No information & $\begin{array}{l}\text { No information on which to base a judgement } \\
\text { about risk of bias }\end{array}$ & $\begin{array}{l}\text { There is no clear indication that the study is at } \\
\text { serious or critical risk of bias, and there is a lack of } \\
\text { information in one or more key domains of bias (a } \\
\text { judgement is required for this) }\end{array}$ \\
\hline
\end{tabular}

Taken from Sterne et al (2016) ${ }^{44}$.

ROBIN-I, Risk of bias in non-randomised studies of interventions. 
Table 3 Interpretation of overall risk-of-bias judgements in ROB2

\begin{tabular}{|c|c|}
\hline Judgement & Criterion \\
\hline Low risk of bias & The study is judged to be at low risk of bias for all domains for this result \\
\hline Some concerns & $\begin{array}{l}\text { The study is judged to raise some concerns in at least one domain for this result, but not to be at high risk } \\
\text { of bias for any domain }\end{array}$ \\
\hline High risk of bias & $\begin{array}{l}\text { The study is judged to be at high risk of bias in at least one domain for this result, or the study is judged to } \\
\text { have some concerns for multiple domains in a way that substantially lowers confidence in the result }\end{array}$ \\
\hline
\end{tabular}

Taken from Sterne et al (2019) ${ }^{45}$

ROB2, Cochrane risk-of-bias tool for randomized trials.

and only RCTs categorised as 'low risk of bias' or 'some concerns' (ROB2) ${ }^{45}$ will be included in the meta-analysis. A systematic narrative synthesis will be provided, and a summary of the characteristics and findings in the studies will be presented in the text and tables. Subgrouping will be used, as appropriate, to ensure clarity of data analysis and presentation of results. Possible subgroupings may include different pain paradigms, motor training paradigms or the presence of statistical heterogeneity. Both behavioural performance measures and activitydependent plasticity outcome measures will be included in the synthesis.

\section{Meta-biases}

Exploring reporting bias is an important part of a systematic review. This will be achieved by undertaking a search of unpublished studies. This will include accessing past conference proceedings of the last 10 years, for example, advances in motor learning and control, pain and progress in motor control and comprehensive internet searches. Study protocols and resultant published studies will be scrutinised to assess for consistencies.

\section{Confidence in cumulative evidence}

To aid the communication of the results of this systematic review, the Grading of Recommendations Assessment, Development and Evaluation (GRADE) approach will be used. ${ }^{46}$ The GRADE approach suggests reporting on both the size of the effect and certainty of evidence. Reporting will use statements recommended by the GRADE working group. ${ }^{47}$ The size of effect will be reported using four categories: large effect; moderate effect, small important effect; and trivial, small unimportant effect or no effect. Similarly, the four categories for certainty of evidence will be high, moderate, low and very low. The quality of evidence will be assessed for each of the individual primary outcome measures included in the PICOS. ${ }^{48}$ This review includes both RCTs and non-randomised studies. As per guidelines around assessing certainty of evidence, initial assessment will begin by classifying the study design. If relevant studies are RCTs, the body of evidence begins as high certainty, whereas for non-randomised studies, the body of evidence will be considered as low certainty ${ }^{49}$ Ratings can then be lowered or raised based on further assessment of eight further domains. Risk of bias, inconsistency, indirectness, imprecision and publication bias are reasons for lowering quality of evidence. Conversely, large effect size, doseresponse gradient and plausible confounding biases that underestimate the effect size are reasons to upgrade the certainty of evidence. ${ }^{50}$

\section{Patient and public involvement}

The research question in this study forms part of a larger discussion within our patient and public involvement meetings. Patients and the public will not be involved in the data collection or data analysis of the review.

\section{Clinical implications}

Pain demands action. In acute pain, this action is primarily protective such as seeking relief. ${ }^{2}$ The resultant protective behaviour may impact on non-pain-related functional goals. ${ }^{3}$ Disruption of non-pain-related functional goals can change our exposure to the environment. Limiting exposure to external stimuli can limit learning or adaptation, an intrinsic component of living. This could include learning to respond to threat or social cues or learning how to perform a specific functional skill. Skill learning in the presence of pain is common in society. Motor skill learning is used regularly in rehabilitation for individuals in pain. ${ }^{51-53}$ The results of studies exploring the impact of pain on motor learning remain conflicting, and this may be due to factors that influence pain experience such as attention, cognition and motivation. This systematic review will provide insights into the interference of pain on motor learning and discuss characteristics of pain experience and of motor skill learning that may influence such interference. This may guide clinicians in the most effective approaches to motor skill learning for individuals experiencing pain.

\section{Ethics and dissemination}

No research ethics is required since no patient data will be collected. Results of this review will be submitted to be published in a peer-reviewed journal and presented at conferences.

\section{Protocol amendments}

Where amendments to the protocol are required, the date and a description and rationale for the changes will be documented.

Twitter Deborah Falla @Deb_Falla 
Contributors Author contributions: DM, EEC, AK and DF were responsible for the conception of the research question, development of the protocol and drafting of the manuscript. DM and EEC will act as first and second reviewers. DM will complete searches and retrieve full-text manuscripts. AK will be the third reviewer. All authors have approved the final manuscript and will contribute to data interpretation, conclusions and dissemination.

Funding The authors have not declared a specific grant for this research from any funding agency in the public, commercial or not-for-profit sectors.

Competing interests None declared.

Patient consent for publication Not required

Provenance and peer review Not commissioned; externally peer reviewed.

Supplemental material This content has been supplied by the author(s). It has not been vetted by BMJ Publishing Group Limited (BMJ) and may not have been peer-reviewed. Any opinions or recommendations discussed are solely those of the author(s) and are not endorsed by BMJ. BMJ disclaims all liability and responsibility arising from any reliance placed on the content. Where the content includes any translated material, BMJ does not warrant the accuracy and reliability of the translations (including but not limited to local regulations, clinical guidelines, terminology, drug names and drug dosages), and is not responsible for any error and/or omissions arising from translation and adaptation or otherwise.

Open access This is an open access article distributed in accordance with the Creative Commons Attribution Non Commercial (CC BY-NC 4.0) license, which permits others to distribute, remix, adapt, build upon this work non-commercially, and license their derivative works on different terms, provided the original work is properly cited, appropriate credit is given, any changes made indicated, and the use is non-commercial. See: http://creativecommons.org/licenses/by-nc/4.0/.

\section{ORCID iDs}

David Matthews http://orcid.org/0000-0003-2687-9132

Edith Elgueta Cancino http://orcid.org/0000-0003-4439-7305

Deborah Falla http://orcid.org/0000-0003-1689-6190

Ali Khatibi http://orcid.org/0000-0003-0679-0499

\section{REFERENCES}

1 Raja SN, Carr DB, Cohen M, et al. The revised international association for the study of pain definition of pain: concepts, challenges, and compromises. Pain 2020;161:1976-82.

2 Tabor A, Van Ryckeghem DML, Hasenbring MI. Pain unstuck: the role of action and motivation. Clin J Pain 2020;36:143-9.

3 Schrooten MGS, Van Damme S, Crombez G, et al. Nonpain goal pursuit inhibits attentional bias to pain. Pain 2012;153:1180-6.

4 Gatzounis R, Crombez G, S Schrooten MG, et al. A break from pain! interruption management in the context of pain. Pain Manag 2019;9:81-91.

5 Vos T, Abajobir AA, Abate KH, et al. Global, regional, and national incidence, prevalence, and years lived with disability for 328 diseases and injuries for 195 countries, 1990-2016: a systematic analysis for the global burden of disease study 2016. The Lancet 2017;390:1211-59.

6 Gatzounis R, Schrooten MGS, Crombez G, et al. Interrupted by pain: an anatomy of pain-contingent activity interruptions. Pain 2014;155:1192-5.

7 Gatzounis R, Schrooten MGS, Crombez G, et al. Activity interruptions by pain impair activity resumption, but not more than activity interruptions by other stimuli: an experimental investigation Pain 2018:159:351-8.

8 Hodges PW, Tucker K. Moving differently in pain: a new theory to explain the adaptation to pain. Pain 2011;152:S90-8.

9 Coombes SA, Misra G. Pain and motor processing in the human cerebellum. Pain 2016;157:117-27.

10 Boudreau S, Romaniello A, Wang K, et al. The effects of intra-oral pain on motor cortex neuroplasticity associated with short-term novel tongue-protrusion training in humans. Pain 2007;132:169-78.

11 Siebner HR, Rothwell J. Transcranial magnetic stimulation: new insights into representational cortical plasticity. Exp Brain Res 2003;148:1-16.

12 Tracey I, Mantyh PW. The cerebral signature for pain perception and its modulation. Neuron 2007;55:377-91.

13 Wager TD, Atlas LY, Lindquist MA, et al. An fMRI-based neurologic signature of physical pain. N Engl J Med 2013;368:1388-97.

14 Apkarian AV, Bushnell MC, Treede R-D, et al. Human brain mechanisms of pain perception and regulation in health and disease. Eur J Pain 2005;9:463-84.
15 Misra G, Coombes SA. Neuroimaging evidence of motor control and pain processing in the human Midcingulate cortex. Cereb Cortex 2015;25:1906-19.

16 Coombes SA, Wang W-E, Roy A, et al. Neurophysiological evidence of the dynamic and adaptive pain-motor interaction. J Physiol 2018;596:2639-40.

17 Ungerleider LG, Doyon J, Karni A. Imaging brain plasticity during motor skill learning. Neurobiol Learn Mem 2002;78:553-64.

18 Doyon J, Albouy G, Vahdat S. Neural correlates of motor skill acquisition and consolidation. In: Brain mapping, 2015: 493-500.

19 Doyon J, Gabitov E, Vahdat S, et al. Current issues related to motor sequence learning in humans. Curr Opin Behav Sci 2018;20:89-97.

20 Tsao H, Galea MP, Hodges PW. Driving plasticity in the motor cortex in recurrent low back pain. Eur J Pain 2010;14:832-9.

21 Vahdat S, Lungu O, Cohen-Adad J, et al. Simultaneous BrainCervical cord fMRI reveals intrinsic spinal cord plasticity during motor sequence learning. PLoS Biol 2015;13:e1002186.

22 Karni A, Meyer G, Jezzard P, et al. Functional MRI evidence for adult motor cortex plasticity during motor skill learning. Nature 1995;377:155-8.

23 Macerollo A, Brown MJN, Kilner JM, et al. Neurophysiological changes measured using somatosensory evoked potentials. Trends Neurosci 2018;41:294-310.

24 Crown ED, Ferguson AR, Joynes RL, et al. Instrumental learning within the spinal cord: IV. induction and retention of the behavioral deficit observed after noncontingent shock. Behav Neurosci 2002;116:1032-51.

25 Ferguson AR, Crown ED, Grau JW. Nociceptive plasticity inhibits adaptive learning in the spinal cord. Neuroscience 2006;141:421-31.

26 Bouffard J, Bouyer LJ, Roy J-S, et al. Tonic pain experienced during locomotor training impairs retention despite normal performance during acquisition. J Neurosci 2014;34:9190-5.

27 Doyon J, Benali $\mathrm{H}$. Reorganization and plasticity in the adult brain during learning of motor skills. Curr Opin Neurobiol 2005;15:161-7.

28 Ingham D, Tucker KJ, Tsao $\mathrm{H}$, et al. The effect of pain on traininginduced plasticity of the corticomotor system. Eur $\mathrm{J}$ Pain 2011;15:1028-34.

29 Bilodeau M-C, Roosink M, Mercier C. Effect of local versus remote tonic heat pain during training on acquisition and retention of a finger-tapping sequence task. Exp Brain Res 2016;234:475-82.

30 Dancey E, Yielder P, Murphy B. Does location of tonic pain differentially impact motor learning and sensorimotor integration? Brain Sci 2018;8. doi:10.3390/brainsci8100179. [Epub ahead of print: 2409 2018].

31 Dancey E, Murphy BA, Andrew D, et al. The effect of local vs remote experimental pain on motor learning and sensorimotor integration using a complex typing task. Pain 2016;157:1682-95.

32 Dancey E, Murphy B, Srbely J, et al. The effect of experimental pain on motor training performance and sensorimotor integration. Exp Brain Res 2014;232:2879-89.

33 Dancey E, Murphy B, Andrew D, et al. Interactive effect of acute pain and motor learning acquisition on sensorimotor integration and motor learning outcomes. J Neurophysiol 2016;116:2210-20.

34 Dancey E, Yielder P, Murphy B. The interactive effect of tonic pain and motor learning on corticospinal excitability. Brain Sci 2019;9. doi:10.3390/brainsci9030063. [Epub ahead of print: 1603 2019].

35 Mavromatis N, Neige C, Gagné M, et al. Effect of experimental hand pain on training-induced changes in motor performance and corticospinal excitability. Brain Sci 2017;7. doi:10.3390/ brainsci7020015. [Epub ahead of print: 0402 2017].

36 Shamseer L, Moher D, Clarke M, et al. Preferred reporting items for systematic review and meta-analysis protocols (PRISMA-P) 2015 elaboration and explanation. BMJ 2015;350:g7647.

37 Moher D, Shamseer L, Clarke M, et al. Preferred reporting items for systematic review and meta-analysis protocols (PRISMA-P) 2015 statement. Syst Rev 2015;4:9.

38 Willingham DB, Nissen MJ, Bullemer P. On the development of procedural knowledge. J Exp Psychol Learn Mem Cogn 1989;15:1047-60.

39 Lamothe M, Roy J-S, Bouffard J, et al. Effect of tonic pain on motor acquisition and retention while learning to reach in a force field. PLoS One 2014;9:e99159.

40 Farrah K, Young K, Tunis MC, et al. Risk of bias tools in systematic reviews of health interventions: an analysis of PROSPERO-registered protocols. Syst Rev 2019;8:280.

41 Quigley JM, Thompson JC, Halfpenny NJ, et al. Critical appraisal of nonrandomized studies-A review of recommended and commonly used tools. J Eval Clin Pract 2019;25:44-52.

42 Borenstein M, Hedges LV, Higgins JPT, et al. A basic introduction to fixed-effect and random-effects models for meta-analysis. Res Synth Methods 2010;1:97-111. 
43 Borenstein M, Higgins JPT, Hedges LV, et al. Basics of meta-analysis: $\mathrm{I}^{2}$ is not an absolute measure of heterogeneity. Res Synth Methods 2017;8:5-18.

44 Sterne JA, Hernán MA, Reeves BC, et al. ROBINS-I: a tool for assessing risk of bias in non-randomised studies of interventions. BMJ 2016;355:i4919.

45 Sterne JAC, Savović J, Page MJ, et al. Rob 2: a revised tool for assessing risk of bias in randomised trials. BMJ 2019;366:8.

46 Guyatt G, Oxman AD, Akl EA, et al. Grade guidelines: 1. IntroductionGRADE evidence profiles and summary of findings tables. J Clin Epidemiol 2011;64:383-94.

47 Santesso N, Glenton C, Dahm P, et al. Grade guidelines 26: informative statements to communicate the findings of systematic reviews of interventions. J Clin Epidemiol 2020;119:126-35.

48 Guyatt G, Oxman AD, Sultan S, et al. Grade guidelines: 11. making an overall rating of confidence in effect estimates for a single outcome and for all outcomes. J Clin Epidemiol 2013;66:151-7.
49 Schünemann HJ, Cuello C, Akl EA, et al. Grade guidelines: 18. How ROBINS-I and other tools to assess risk of bias in nonrandomized studies should be used to rate the certainty of a body of evidence. $J$ Clin Epidemiol 2019;111:105-14.

50 Balshem H, Helfand M, Schünemann HJ, et al. Grade guidelines: 3. rating the quality of evidence. J Clin Epidemiol 2011;64:401-6.

51 van Dieën JH, Flor H, Hodges PW. Low-Back pain patients learn to adapt motor behavior with adverse secondary consequences. Exerc Sport Sci Rev 2017;45:223-9.

52 Tsiringakis G, Dimitriadis Z, Triantafylloy E, et al. Motor control training of deep neck flexors with pressure biofeedback improves pain and disability in patients with neck pain: a systematic review and meta-analysis. Musculoskelet Sci Pract 2020;50:102220.

53 Ravichandran H, Janakiraman B, Gelaw AY, et al. Effect of scapular stabilization exercise program in patients with subacromial impingement syndrome: a systematic review. J Exerc Rehabil 2020;16:216-26. 